\title{
Self Magnetic Flux Leakage as a Diagnostic Signal in the Assessment of Active Stress - Analysis of Influence Factors
}

\author{
M. Roskosz*, A. ZŁOCKI And J. KwaŚNIEWski \\ AGH University of Science and Technology, Department of Mechanical Engineering and Transport, \\ Faculty of Mechanical Engineering and Robotics, al. A. Mickiewicza 30, 30-059 Kraków, Poland
}

\begin{abstract}
On the macroscopic scale, mechanical and magnetic properties of ferromagnetics are closely related. Under mechanical stress, ferromagnetics are subject to deformations alongside the changes in magnetization. Stresses and strains lead to changes of interaction forces acting between adjacent atoms and lead to a change in the magnetization distribution in domains, which in turn results in a change of the magnetic properties. Generally, these phenomena are referred to as magnetomechanical effects. As a part of this research study, specimens of P91 steel were tested after being subjected to diverse heat treatment procedures affecting their microstructure. The samples were subjected to periodically pulsed tensile cyclic loads with different values of the active stress induced in the part of the sample used in the measurements. For each of the tested combinations of applied stresses and microstructures, the magnetization changes followed a different pattern although certain similarities were reported, too. The greatest changes in the value of the sample magnetization were registered in the initial load cycles. There were significant differences in the magnetization process between samples with a different microstructure. Potential applications of the self-magnetic flux leakage value to analyses of active stresses in the sample were explored.
\end{abstract}

DOI: 10.12693/APhysPolA.137.690

PACS/topics: P91 steel, measurement of self magnetic flux leakage, magnetization, microstructure

\section{Introduction and aims of the study}

Experimental and theoretical determination of magnetization versus active and residual stresses while taking into account the microstructure of the sample, the result of heat treatment, is one of the fundamental tasks of magnetic studies. This issue is highlighted in the basic model of the Jiles-Atherton-Sablik [1,2] with later extensions [3]. From the standpoint of potential applications, the correlation between magnetization and active stress, which in the present case is restricted to the correlation between self magnetic flux leakage (SMFL) measured on the surface of the sample and active stresses, can be used for identification of stress and strain state inverse problems in non-destructive testing [4-10]. This study aims at a qualitative and quantitative analysis of magnetization versus periodically pulsed tensile cyclic loads with different values of the active stress acting upon the P91 steel samples subjected to various heat treatment processes. P91 boiler steel (X10CrMoVNb9-1) is increasingly used in the power industry in the form of pipes, sheets, forgings, and bars, replacing standard grades such as $13 \mathrm{HMF}$ and $14 \mathrm{MoV} 6-3$. It is categorized as high-alloy and high-temperature boiler steel because of increased content of chromium and small amounts of less frequently used alloy additives.

*corresponding author; e-mail: mroskosz@agh.edu.pl

\section{Experimental procedure}

Testing was done on plate samples of P91 steel (X10CrMoVNb9-1) with a narrowed section in the middle part. The samples were subjected to various heat treatment processes. Sample 1 is the steel in delivery state, its microstructure composed of highly dispersed martensite with numerous coagulated fine carbides and few small irregular ferrite area, with revealed visible grain boundaries of primary austenite. Sample 2 is obtained after oil quenching from $1060^{\circ} \mathrm{C}$ (furnace, air) and after tempering at $750{ }^{\circ} \mathrm{C}$ (furnace, air). Its microstructure is composed of martensite with a small amount of residual austenite and irregular fine ferrite areas with visible grain boundaries of primary austenite. Sample 3 is normalized at $1060{ }^{\circ} \mathrm{C}$ (furnace, air), and then cooled in the furnace. Its microstructure is fine ferrite grains with numerous fine coagulated carbides, also spotting few fine ferrite grains without precipitates and ferrite areas along grain boundaries and beyond.

Each sample was subjected to 10 cycles of pulsating tensile stress loads with a maximum load value applied in a smaller sample cross-section with the maximum stresses of the cycles of 100, 200, and $400 \mathrm{MPa}$, respectively. Before the tests, the samples were demagnetized. This process was repeated before each increase in the maximum tensile force.

SMFL measurements in the middle regions of the tested sample were taken with a SpinMeter3D sensor manufactured by "Micro Magnetics Sensible Solutions". The measuring range of the sensor is $\pm 1000 \mu \mathrm{T}$, affording the sensitivity of $0.1 \mu \mathrm{T} \approx 0.08 \mathrm{~A} / \mathrm{m}$. The sensor was 
duly calibrated in a tube made from Mu-Metal, which ensures a high damping level, and inside which the magnetic field strength becomes zero. The sensor was placed with $3 \mathrm{~mm}$ lift-off on one side of the sample positioned in the cylinder head in order to measure the magnetic field in the middle point of the sample.

Measurements were taken for three components of SMFL on the surface of the samples: $H_{x}$ - tangential component measured in the direction parallel to the load applied, $H_{y}$ - tangential component measured in the direction perpendicular to the load applied, $H_{z}$ - normal component. The triaxial magnetometer reads off other diagnostic symptoms: magnitude of the magnetic field vector, angle of deviation of the vector's tangent component from the axis of the specimen $\Phi_{1}$, the inclination angle of the vector with respect to the sample surface $\Phi_{2}$.

\section{Measurement results and analysis}

According to the model of the Jiles-AthertonSablik [1, 2], the change in magnetization $M$ due to stress can be described by the following equation:

$$
\frac{\mathrm{d} M}{\mathrm{~d} \sigma}=\frac{\sigma}{E \xi}(1-c)\left(M_{\mathrm{a}}-M_{\mathrm{irr}}\right)+c \frac{\mathrm{d} M_{a}}{\mathrm{~d} \sigma},
$$

where $\xi$ is a relaxation factor, $c$ is material constant, $E$ is Young's modulus, $M_{\text {irr }}$ is irreversible magnetization, and $M_{\mathrm{a}}$ is an anhysteretic magnetization.

The intensity of RMF near the ferromagnetic object can be expressed by

$$
\boldsymbol{H}(\boldsymbol{r})=\boldsymbol{H}_{a}(\boldsymbol{r})+\boldsymbol{H}_{d}(\boldsymbol{r}),
$$

where $H_{a}$ is external magnetic field strength, and $H_{d}$ is magnetic field strength associated with magnetization of the ferromagnetic. $H_{d}$ is referred to as the demagnetization field and it is described by the equation

$$
\begin{gathered}
\boldsymbol{H}_{d}(\boldsymbol{r})=\frac{1}{4 \pi} \int_{V} \frac{-\boldsymbol{\nabla} \cdot \boldsymbol{M}(\boldsymbol{s})}{|\boldsymbol{r}-\boldsymbol{s}|^{3}}(\boldsymbol{r}-\boldsymbol{s}) \mathrm{d} V(\boldsymbol{s}) \\
+\frac{1}{4 \pi} \int_{S} \frac{\boldsymbol{n} \cdot \boldsymbol{M}(\boldsymbol{s})}{|\boldsymbol{r}-\boldsymbol{s}|^{3}}(\boldsymbol{r}-\boldsymbol{s}) \mathrm{d} S(\boldsymbol{s}),
\end{gathered}
$$

where $\boldsymbol{M}$ is magnetization, $V$ is the volume, $S$ is the surface area of the ferromagnetic, and $s$ is the vector plotted for any inner point or a point on the surface of the magnetometer, $\boldsymbol{r}$. The values and distribution of magnetization $\boldsymbol{M}(s)$ results in a unique distribution and values of magnetic field strength $\boldsymbol{H}(r)$.

Time profiles of SMFL were obtained for three components on the surface of the tested samples - selected results are shown in Fig. 1 (sample 2 - maximum stress $400 \mathrm{MPa}$ ). The most significant changes were registered for the tangential component $H_{x}$ measured in the direction parallel to the load applied. In the next part of study, the main focus is on changes in the value of $H_{x}$ component and the value of calculated vector magnitude $H$. These results were correlated with the stress changes and the halves of the magnetomechanical hysteresis loop were derived accordingly. In all next figures, for the first three cycles, the blue color determines the measurements

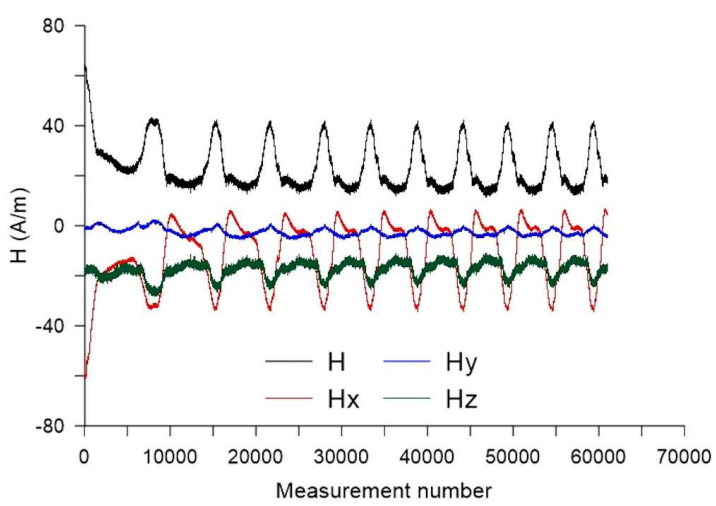

Fig. 1. Time profile of components of SMFL.

obtained during loading the sample and the red color during unloading. The black line is the results for the last 10 cycles of load changes. As a representative of all measurements cycle 0 to $400 \mathrm{MPa}$ was chosen and the results of the measurement are shown in Figs. 2 and 3. Figure $2 \mathrm{a}-\mathrm{c}$ presents relationship between active stress and tangential component parallel to the load applied $H_{x}$ and Fig. $3 \mathrm{a}-\mathrm{c}-$ between stress and magnetic field strength vector module $H$.

The greatest increase in SMFL is registered in the first cycle of loading, and in each subsequent cycle the change decreases. This conclusion concerns mainly irreversible changes in magnetization, with relatively stable reversible changes. After stabilization of the magnetomechanical hysteresis loop, the registered variability of magnetization due to stress variations are mostly reversible changes. The influence of the sample microstructure on magnetization patterns is readily apparent. The magnetization plot for sample 3 with a ferritic microstructure is clearly different from samples 1 and 2 with a dominant martensitic microstructure. Figures 2 and 3 and other test results, not included in this publication, show that for each combination of microstructure and the maximum stress value, the variability pattern of SMFL will be different and so will be its maximum and minimum values. The increase in the maximum cycle stress causes the increase of both maximum and minimum (SMFL) values. The increase in the maximum cycle stress in all samples causes an increase in the maximum values of the $H_{x}$ component, whereas the maximum value $H_{x}$ for the samples with martensitic microstructure is registered for stress levels below the maximum value in the cycle. Analysis of the variability patterns of $H$ (see Fig. 3) reveals that for samples with a martensitic microstructure, the most intensive changes occur in the initial stage of the loading cycle (sample 1: 0-200 MPa, sample 2: 0-100 MPa). For the sample with a ferritic microstructure the most intensive changes occur in the mid-stage of the loading cycle (sample 3: 100-300 MPa). In qualitative terms, the experimental results are consistent with those reported in [3]. Stress-induced variations of the deviation angle of the tangent component of the vector $H$ from the axis of the specimen $\Phi_{1}$ were found to be negligible. 

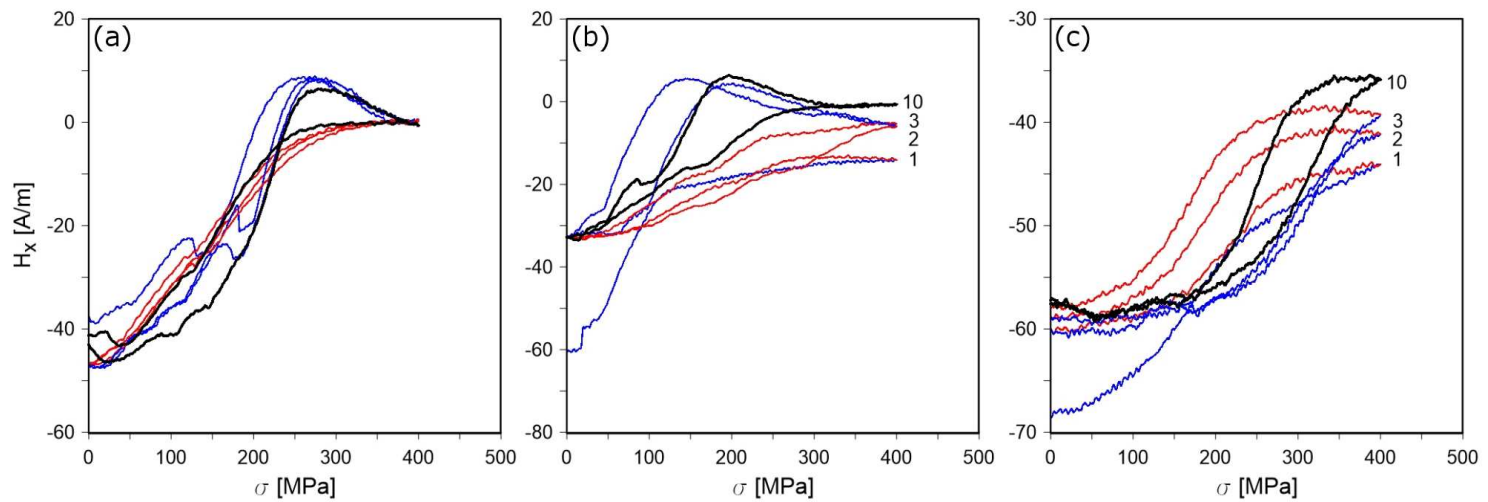

Fig. 2. Half of magneto-mechanical hysteresis loop - tangential component parallel to the load applied $H_{x}$, cycle 0-400 MPa: (a) sample 1, (b) sample 2, (c) sample 3.
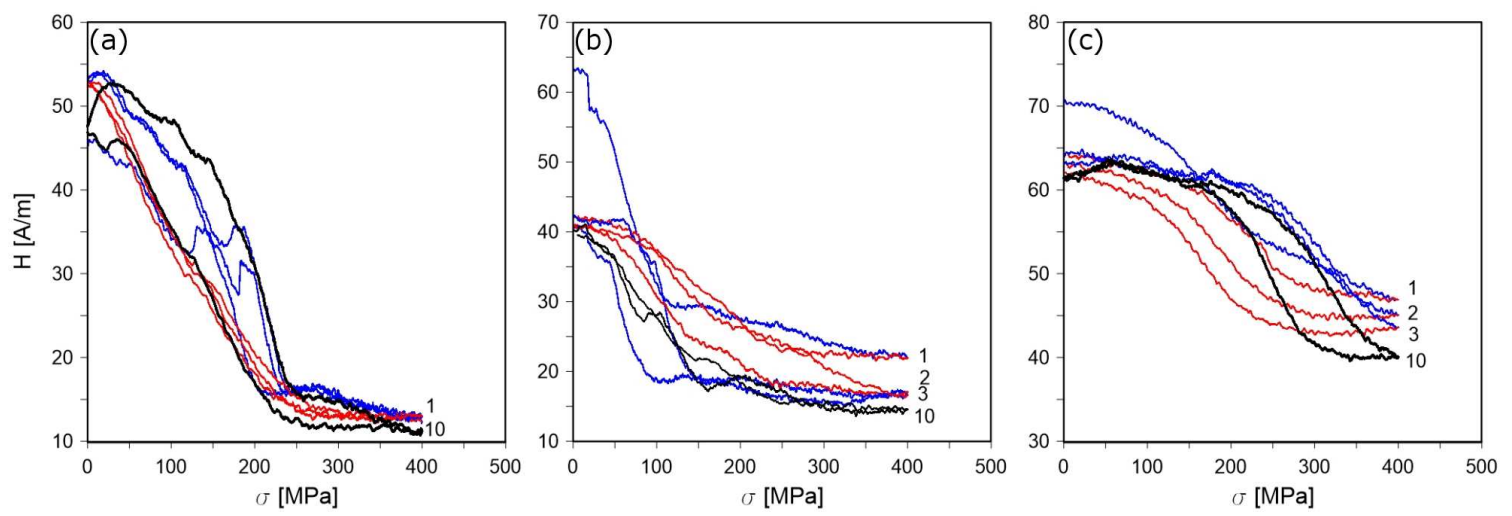

Fig. 3. Half of magneto-mechanical hysteresis loop, magnetic field strength vector magnitude $H$, cycle 0-400 MPa: (a) sample 1, (b) sample 2, (c) sample 3.

Changes of the inclination angle of the vector $H$ to the surface of the sample $\Phi_{2}$ were of the order of several degrees for sample 3 with a ferritic microstructure and in excess of ten degrees for samples 1 and 2, with a martensitic microstructure.

\section{Summary}

Previous studies $[4,5,8,10]$ have shown that when deriving quantitative diagnostic correlations based on measurements of magnetic properties, it is required that a number of impact factors should be taken into account. These include geometry of the element (thickness and influence of magnetic field disturbances near the edge of the element), state of stress (active and residual) acting upon the sample, feasibility of identifying the direction of principal stress and surface condition (roughness, coatings).

The results summarized in this study clearly demonstrate both the influence of the microstructure and the effect of the maximum load cycle stress on the observed changes in SMFL caused by variable loads. The microstructure of the controlled object is rather constant (excluding changes due to thermal, fatigue, and ionizing radiation degradation) and identifiable, yet the full history of the loading process may be unknown. The results lead us to the conclusion that identification of the active stress state in a controlled object on the basis of SMFL measurements may involve certain difficulties when the full history of the loading process is unknown. For that very reason, the quantitative analysis of experimental results could not be conducted.

\section{References}

[1] D.C. Jiles, J. Phys. D Appl. Phys. 28, 1537 (1995).

[2] D.C. Jiles, D.L. Atherton, J. Magn. Magn. Mater. 61, 48 (1986).

[3] L. Li, D.C. Jiles, IEEE Trans. Magn. 39, 3037 (2003).

[4] M. Roskosz, M. Bieniek, Int. J. Appl. Electromagn. Mech. 39, 521 (2012).

[5] R. Stegemann, S. Cabeza, V. Lyamkin, G. Bruno, A. Pittner, R. Wimpory, M. Boin, M. Kreutzbruck, J. Magn. Magn. Mater. 426, 580 (2017).

[6] H. Haihong, Q. Zhengchun, Y. Cheng, H. Gang, L. Zhifeng, Nondestruct. Test. Evaluat. 32, 166 (2016).

[7] N. Venkatachalapathi, S.M.D. Jameelbasha, G. Janardhan Raju, P. Raghavulu, Mater. Today Proc. 5, 8645 (2018).

[8] M. Witoś, M. Zieja, N. Fallahi, Z.H. Żurek, J. Kwaśniewski, Acta Phys. Pol. A 133, 697 (2018).

[9] M. Maciusowicz, G. Psuj, Sensors 19, 1443 (2019).

[10] M. Roskosz, M. Bieniek, NDTEE Int. 54, 63 (2013). 